\title{
¿Inglés bolivariano? ¿Estructuralista o comunicativo? Estudio con libros de educación media de Venezuela (*)
}

\author{
Bolivarian English? Structuralist or Communicative? \\ A study using High School Textbooks from Venezuela
}

ISSN 1510-2432 - ISSN 1688-9304 (en línea) - DOI: https://doi.org/10.18861/cied.2016.7.2.2611

Carlos Eduardo Blanco

Doctor (Cand.) en Educación, Universidad Central de Venezuela. Magíster en Educación, Universidad de Toronto, Canadá. Profesor titular. Centro de Investigaciones Educativas, Universidad Central de Venezuela.

Fecha de recibido: 14/02/2016

Fecha de aceptado: 05/07/2016

\section{Resumen}

Analizar textos escolares puede develar concepciones acerca de destrezas e innovaciones pedagógicas, entre otros fenómenos. Por tal razón se realizó un estudio empírico con libros de la colección estatal venezolana Bicentenario. Los objetivos fueron analizar las actividades de escritura que se promueven en libros de la Educación Media, específicamente de $4 .^{\circ}$ y $5 .^{\circ}$ año, relacionar dichas actividades con teorías actuales acerca del aprendizaje del inglés y averiguar qué tipo de soporte pudieran dar estos libros a los educandos. Los criterios para la selección de los libros fueron: ser de $4 .^{\circ}$ y $5 .^{\circ}$ año, de editorial estatal y de uso corriente en los liceos del país. Se analizaron las actividades de escritura en dos libros. La estrategia de análisis fue emergente pues las categorías fueron suministradas por los textos al interactuar el investigador con ellos. Se abordaron las 317 cláusulas con los 30 verbos rectores de las actividades (write, answer, complete, fill in, entre otros). Los resultados muestran, primero, que ambos libros dan importancia a la destreza de la escritura. Segundo, que si bien no se apartan completamente del paradigma estructuralistaconductista, sí intentan promover la comunicación escrita, con equilibrio de actividades, lo cual pudiera dar soporte a clases menos tradicionales.

Palabras clave: libros de texto, inglés, escritura, educación secundaria, Venezuela.

\begin{abstract}
The analysis of school texts can reveal conceptions about language skills and educational innovations, among other phenomena. For such reason, an empirical study was conducted using books of Venezuela's state-edited collection Bicentenario. The objectives of the study were to analyze the writing activities promoted in upper high school English books (fourth and fifth grades specifically), to relate such activities with current theories about the learning process of English as a foreign language and to find out what kind of support these books might provide to students. The criteria for book selection were as follows: to belong to the last two years of high school, to be edited by the Ministry of Education, and to be currently under use at high school institutions. The writing activities in two books were analyzed. The strategy of analysis was emergent for the categories were provided by the texts as the researcher interacted with them. The 317 clauses along with 30 verbs (write, answer, complete, and fill in, among others) leading the activities were approached. Results show, in the first place, that both books considered writing to be an important skill. The second conclusion is that, despite books do not depart completely from the structuralist-behaviorist
\end{abstract}


paradigm, they do try to promote communicative writing, with balanced activities and in this way, less traditional class work would be supported.

Keywords: textbooks, English, writing, secondary school, Venezuela.

\section{Consideraciones teóricas}

El presente estudio parte de varios hechos e hipótesis aceptadas. En primer lugar, que existe un área problemática de la actividad del sistema de educación formal de Venezuela: la enseñanza del inglés. La misma se inicia con la Capitanía General de Venezuela en 1777, primordialmente en la Universidad de Caracas, fundada en 1721 (Leal, 1981). A comienzos del siglo XX funcionaba la Escuela Nocturna de Inglés de Caracas (Baptista, 1910) y en los colegios de niñas se estudiaba el inglés durante los tres años de educación (Gil Fortoul, 1912). Con la reforma del sistema escolar venezolano de 1914, en el bachillerato de Filosofía y Letras se incluía el inglés para $3 .^{\circ}$ y $4 .^{\circ}$ año (Guevara Rojas, 1915). Con la creación del Pedagógico Nacional en 1936 se impulsan los estudios académicos de las materias que se impartían en el bachillerato (Hernández, 2012). En segundo lugar, que el tan importante tema de los textos escolares (Apple, 1984) no ha sido documentado para el área de idioma inglés de educación media en este país. En tercer lugar, que para algunos (Castellanos, 2011) Venezuela vive a comienzos del siglo XXI un momento histórico excepcional y los libros de texto escolares podrían recoger huellas de ese devenir (Bloch, 1979) tales como concepciones acerca de los cursos en ediciones estatales, entre otras. En cuarto lugar, que los textos escolares suelen incluir un componente práctico que se materializa en actividades, las cuales a su vez frecuentemente son vehiculadas mediante instancias del lenguaje escrito, por medio de estructuras lingüísticas (Johnsen, 1996).

Por una parte, desde tiempos inmemoriales la escritura y la educación han ido juntas (Webb, Metha y Jordan, 1996). En la enseñanza del inglés ello no ha sido excepción desde el inicio de esta profesión en el siglo XVI (Howatt, 1984) aunque la escritura del idioma haya cobrado mayor relevancia en las últimas décadas (Hyland, 2003). La escritura ha sido considerada una de las cuatro destrezas básicas del idioma y las actividades de los cursos son tal vez el componente más revelador de la enseñanza de cualquier materia o destreza (Chaudron, 1998).

Por otra parte, es aceptado que las concepciones acerca de la escritura pueden cambiar, aunque también mantenerse (Raimes, 1983; 1998) mientras que los libros de texto pueden incluir concepciones (Herrera, 2012; Lopes, 2015; Rodrigues y Marques da Fonseca, 2014; Varón, 2009; Zapata, 2011) no siempre explícitas en los contenidos. Escudriñando los libros se puede llegar a saber no solamente qué se entiende como lo más adecuado o lo más avanzado dentro de un ámbito de conocimientos, sino también qué se está enseñando y por ende qué se está aprendiendo (Wray y Bloomer, 2006). En consecuencia, indagar cómo se plantea la destreza de la escritura -qué actividades se promueven en libros de texto de inglés- puede contribuir con importantes aspectos de la teoría y la práctica del campo de la enseñanza de este idioma, componente importante de la educación a escala mundial.

\subsection{Los textos escolares: géneros de peso}

El presente estudio aborda un aspecto de la relación entre los géneros del lenguaje en tanto creaciones de la cultura y un ámbito de la educación formal como es la enseñanza del inglés. Los géneros son patrones que pueden ser identificados y compartidos por comunidades discursivas, dependen del contexto social en el que se crean y utilizan, se materializan en textos, responden a situaciones de comunicación y reflejan normas de una comunidad así como su epistemología, ideología y ontología social. Desde un punto de vista estructural, se definen como un patrón comunicativo complejo que puede situarse 
en tres niveles: el de la estructura interna, relativa a rasgos verbales y no verbales, el nivel situativo, relacionado con el contexto interactivo, y el de la estructura externa, o ámbito comunicativo y de distribución institucional. Las tendencias en el estudio de los géneros van desde la nueva retórica a la lingüística sistémica funcional, la lingüística aplicada, la semiótica discursiva, la cognición social y la acción social, entre otras y en múltiples ámbitos. Tales enfoques se diferencian en cómo abordan metodológicamente los textos y se asemejan en que se basan en las situaciones comunicativas en las que se producen y se utilizan, así como en sus propósitos comunicativos (Briceño-Velazco, 2014; Medina y Domínguez, 2009).

Dado que se relaciona muy cercanamente con el lenguaje en uso y la comunicación, la temática de los géneros permea y se aplica en los más variados ámbitos de la actividad humana y diferentes áreas del saber, entre ellas la educación. Con relación a esto, Medina y Domínguez (2009) han afirmado que:

“[...] un género discursivo dado lleva consigo implicaciones sociales e históricas que le moldean en tanto que elemento de comunicación y que están sujetas a cambios en la medida en que sus implicaciones culturales cambien [...]". (Medina y Domínguez, 2009: 205)

Por su parte, Ciapuscio (2005) ha señalado el carácter complejo, ideal, potencial y de experiencia social y comunicativa de los géneros. Relacionando específicamente dicho señalamiento con el ámbito de la educación puede destacarse el carácter de funcionalidad de los textos con su finalidad de informar y de dirigir, el carácter de situacionalidad, o de organización social de las actividades, el carácter de tematicidad y estructura, relacionado con los despliegues del tema textual y la estructuración, y el de adecuación de la formulación, o los esquemas de formulación específica del género y sus particularidades estilísticas (Ciapuscio, 2005).

Así entonces, uno de los géneros al que podría clasificarse como un peso pesado es el libro de texto escolar pues la educación formal tal como la conocemos sería impensable sin los libros que instituciones y profesionales asignan a los educandos (Ramírez, 2004). Dichos recursos pedagógicos son el medio en torno al cual suelen desenvolverse gran parte de las actividades del año escolar y en las últimas décadas han suscitado un creciente y justificado interés por su estrecha vinculación con cuestiones de actualidad tales como la complejidad creciente de los manuales, la incidencia de las tecnologías y la formación docente, entre otras. Analizar ediciones escolares en ocasiones implica tomar en cuenta el contexto nacional pues la literatura escolar se concibe como la expresión de una identidad y el medio para crearla, lo que explica en buena medida las polémicas de las que es objeto con frecuencia el texto escolar (Choppin, 2000).

Los factores que influyen en el texto escolar están frecuentemente relacionados con los modos de financiación, los procedimientos de control, las técnicas de fabricación, las reales o pretendidas innovaciones pedagógicas, entre otros, y que se manifiestan de forma más evidente en el contenido intelectual (traducciones, adaptaciones, préstamos) o en la forma misma de las obras (organización o estructura interna). Estos factores son muestras de la circulación de capitales, ideas, contenidos o métodos, por lo que emprender estudios con textos escolares supone necesariamente, a nivel nacional o regional, censar la producción, localizar colecciones, recopilar textos reglamentarios e inventariar empresas de edición, entre otras actividades (Choppin, 2000). 
Por tanto, al referirnos al libro de texto escolar hablamos de uno de los componentes más emblemáticos de la cultura y la actividad de la enseñanza, considerado en gran medida estratégico, razón por la cual ha sido abordado por estudiosos de la educación (Güemes, 1994; Level y Mostacero, 2011; Puéllez y Tiana, 2003; Tosi, 2011), ya sea asumido como objeto o producto de consumo, como soporte de los conocimientos para los educandos, como vector ideológico y cultural o como instrumento pedagógico. Al libro de texto escolar lo definiremos en términos de Ramírez (2004: 34) como:

“[...] un recurso didáctico, que puede ser de sustrato material o virtual, en el cual se materializa un discurso compuesto por palabras, palabras y símbolos o palabras, símbolos e ilustraciones; estructurado de manera secuencial y sistemática en atención a la maduración intelectual y emocional del lector, y creado con la intención expresa de ser utilizado como un recurso pedagógico en el proceso de enseñanza-aprendizaje del sistema escolar formal, con el fin de brindar información sobre algún área del conocimiento en atención a la oferta curricular establecida en los programas de estudio elaborados por las autoridades educativas nacionales, quienes a su vez autorizan, supervisan y reglamentan sus contenidos, extensión y tratamiento".

En atención a lo anterior, se ve justificado el análisis de textos escolares, por ejemplo, los utilizados para impartir cursos de idioma inglés a quienes no lo tienen como lengua materna. Un estudio como el presente, que analiza dos libros de inglés en cuanto a componentes de su organización o estructura interna (actividades de escritura) en su relación con posibles innovaciones pedagógicas (teorías actuales) y también en tanto soporte de los conocimientos para los educandos (combinación de esos dos análisis) puede ser de mucho interés para docentes e investigadores del campo del idioma inglés para hablantes de otras lenguas, para instituciones formadoras, hacedores de política educativa y editores, entre otros.

\subsection{La enseñanza del inglés. Latinoamérica y Venezuela}

La enseñanza del idioma inglés es una empresa gigantesca, multimillonaria, que se fundamenta en la alta demanda de dicho recurso lingüístico para comunicar -para los más diversos fines y propósitos- a centenares de millones de personas que no comparten un idioma materno común (Brown, 2007; Gregson, 2006; Zeuch y Gregson, 2015). Para Díaz Larenas et al. (2013) la enseñanza y el aprendizaje del inglés en los países iberoamericanos han estado históricamente marcados por enfoques lingüísticos y pedagógicos provenientes inicialmente del enciclopedismo europeo y el conductismo estadounidense, corrientes que con frecuencia definieron lo que significaba dominar una lengua extranjera. El enfoque de gramática y traducción promovía, por ejemplo, la enseñanza de estructuras lexicales y sintácticas que, en conjunto con la estrategia de traducción permitirían, en teoría, dominar el idioma. Posteriormente, el conductismo propiciaría el enfoque audiolingual, caracterizado por exponer al estudiante a una serie de prácticas lingüísticas de modelamiento y repetición con el fin de que alcanzara un nivel de comprensión auditiva y expresión oral similar al de un angloparlante.

Con el advenimiento del enfoque comunicativo en los años setenta del siglo XX -una revolución científica en su campo (Raimes, 1983; Savignon, 2005)- la enseñanza de lenguas se orienta hacia el desarrollo de un constructo como la competencia comunicativa, es decir: aprender una lengua es comunicarse e interactuar con los demás (Díaz Larenas et al., 2013). Las aulas latinoamericanas, con más o menos recursos, han estado centradas en tratar de lograr que el estudiante sea capaz de escuchar, leer, escribir y hablar una lengua extranjera y comprender los aspectos culturales esenciales que condicionan el 
dominio de una lengua que no es la propia (Díaz Larenas et al., 2013). Venezuela, por su parte, no ha estado ajena a tal proceso y ha transitado por diferentes enfoques didácticos relativos a la enseñanza del inglés, fundamentalmente a nivel secundario y universitario, así como a la formación de profesores (López de D’Amico, 2015, 2010; Mota, 2006). En este país se cuenta con los programas de estudio oficiales de la Educación Primaria y de la Educación Media, fundamentados en el Diseño Curricular del Sistema Educativo Bolivariano y el Currículo del Subsistema de Educación Secundaria Bolivariana, ambos de 2007 (Beke, 2015). Asimismo, se cuenta con los estándares lingüísticos y pedagógicos de las instituciones universitarias formadoras.

López de D'Amico (2010) ha caracterizado la enseñanza del inglés en Venezuela describiéndola como productora de bachilleres desmotivados, con bajo dominio y escasos conocimientos del idioma. Una actividad en la que se utiliza el libro de texto como recurso casi único, donde hay necesidad de innovar, donde se evidencia la falta de evaluación de los métodos de enseñanza, el aislamiento de los docentes y la incongruencia entre los objetivos y los materiales, situación que se ha venido manifestando desde hace décadas. Dicha autora señala que la importancia de la enseñanza del inglés se reconoce por lo menos en el papel, aunque se mantiene actualmente como asunto problemático y como reto académico. Por su parte, para Hernández (2012), en Venezuela las asignaturas correspondientes a idiomas han cambiado poco, manteniéndose las mismas en el transcurso de las décadas de 1950, 1960 y 1970, cuando el inglés se volvió obligatorio en los liceos. Expresa Hernández (2012) que en la actualidad el Liceo Bolivariano propone una integración de áreas de conocimiento a través de proyectos, y que entre las cinco áreas que integran las disciplinas y contenidos se encuentra la de Lengua-Cultura-Idioma que incluye el inglés, el castellano, el portugués y las lenguas autóctonas.

En cuanto a la enseñanza del inglés en la educación secundaria pública en Venezuela, Beke (2015) evidenció asuntos por atender. Por ejemplo, no está bien establecido el cuánto y el cómo del uso de los libros de texto en las aulas. En la actividad de los docentes prevalece una concepción estructuralista o tradicional en la que la gramática es lo más importante, con bajo nivel de comunicación en la lengua extranjera (Castillo, Gamero y Sivira, 2015). La colección estatal de libros Bicentenario no incluyó los textos de inglés sino hasta finales de 2013, cuando comenzaron a distribuirse en los planteles. Así, es poco lo que se sabe acerca del verdadero uso del texto de inglés en las aulas venezolanas (Beke, 2015), de modo que acaso pudiera hipotetizarse que los libros de distribución gratuita aquí analizados sean utilizados mayormente en el sector de la educación estatal, que atiende a la mayoría de los estudiantes del país. Finalmente, otro aspecto que cabe señalar es que desde 1998, y hasta el momento de escribir este artículo, no se conocen los resultados de las evaluaciones de los aprendizajes del sistema escolar venezolano realizadas mediante estudios oficiales. Sin embargo, según una evaluación privada efectuada recientemente, Venezuela figura entre los últimos en cuanto a dominio del inglés, pues entre 63 países el país fue clasificado en el número 50, en nivel muy bajo (5. a categoría) (English First, 2014; Villar, 2012).

\subsection{La escritura en inglés y la educación media venezolana}

La escritura es el acto o proceso de producir y registrar palabras de forma que puedan ser leídas y comprendidas. Específicamente en la enseñanza de lenguas extranjeras la expresión escrita es una de las cuatro destrezas comunicativas. En la enseñanza del inglés para no nativos se le considera uno de los más exigentes componentes del aprendizaje, habilidad compleja que requiere de instrucción especializada y práctica extensiva, la que ha llegado a asumir una posición cada vez más central en los programas de estudio, mucho más de la que tenía una o dos generaciones atrás. Según Hyland (2003) la escritura no 
puede circunscribirse a un conjunto de habilidades cognitivas o técnicas, o a un sistema de reglas. Tampoco se trata de una cuestión de oportunidades para componer y revisar, pues los educandos traen al aula conocimientos para crear textos efectivos y tales conocimientos deben ser tenidos en cuenta en la enseñanza.

Desde hace varias décadas se ha abordado el complejo asunto de la escritura del inglés como lengua extranjera, se han clasificado los tipos de escritura y se ha tratado de informar sobre las prácticas de los salones de clase (Mota, 2006). Tales trabajos han fundamentado movimientos que han evolucionado históricamente, pero sin que uno haya necesariamente reemplazado completamente al anterior. Por ejemplo, Hyland (2003) ha clasificado dichas opciones como aquellas organizadas en torno a estructuras lingüísticas, funciones textuales, temas o tópicos, expresión creativa, procesos de composición, contenidos y géneros y contextos de la escritura. Mas la tendencia ha sido a tener en cuenta un continuum que va desde lo más restringido hasta lo más libre, lo más estructural hasta lo más funcional y creativo, donde, por ejemplo, la opción de enseñanza más tradicional, basada en estructuras lingüísticas, suele implicar una secuencia de familiarización, escritura controlada, escritura guiada y escritura libre.

Para Mota (2006) la escritura en el sistema escolar venezolano no puede concebirse solo como un instrumento que ayude a los estudiantes a aprender la lengua y a procesar el contenido de un curso, sino que también es una herramienta para desarrollar conocimiento.

Para ello, el dominio de la lengua materna es esencial, pero la escritura es una destreza no bien atendida y que no debe postergarse dentro del currículo (Mota, 2006). Por su parte, Beke (2015) halló una situación poco halagadora en cuanto al inglés en liceos venezolanos en relación con los actores del proceso de enseñanza, el programa que se sigue, la concepción de aprendizaje del idioma y la manera en que se enseña y evalúa. Esta autora no abordó directamente la enseñanza de la escritura del inglés pero sí aportó hallazgos relacionados, tales como falta de fundamentación en la política referida a recursos y materiales de enseñanza, poca claridad en cuanto a qué programa se utiliza, existencia de -si acaso- una concepción tradicional de enseñanza y no obligatoriedad de la adquisición de libros de inglés.

\subsection{Textos escolares de inglés}

Entre las opciones de investigación de los profesionales de la educación se encuentran los textos escolares. En Venezuela han sido objeto de indagación casi exclusivamente en los niveles de educación primaria y básica (Arteaga y Alemán, 2007; Beyer, 2011; Bisbe, 2009; Level y Mostacero, 2011; Mujica, Díaz y Arnáez, 2008; Ramírez, 2004) en las áreas de sociales, matemática y lengua. Con referencia a los de idioma inglés, Zapata (2011) abordó los tipos y niveles de estrategias de comprensión de la lectura en libros de la carrera de inglés en la Educación Superior. En otros países de la subregión latinoamericana, Herrera (2012), Lopes (2015) y Varón (2009) han investigado libros de inglés en cuanto a actividades y potencial para promover aprendizaje, así como conceptos y teorías implícitas. Según estos trabajos, los libros de texto son uno de los componentes principales de la actividad de las aulas de inglés, mientras que para Hyland (2003) los textos comercialmente producidos representan el material más comúnmente utilizado en las clases de escritura de ese idioma alrededor del mundo y seguramente habrá pocos docentes que no hagan uso de ellos.

El estudio aquí reportado analiza libros editados por el sector gubernamental, cuya participación en la edición de textos escolares en Venezuela había sido escasa hasta hace muy poco (Ramírez, 2015, 2007; Level y Mostacero, 2011). No se analizan libros de escritura propiamente sino aquellos que incluyen dentro de sí, junto con el tratamiento de otras 
destrezas como la expresión oral, la comprensión auditiva y la lectura, el componente relativo a la destreza escritural. Las destrezas básicas del idioma están vehiculadas en los textos de manera entremezclada. En primer lugar, según los objetivos de los programas $y$, en segundo lugar, de acuerdo con el conjunto de criterios profesionales y de otra índole que pudieran converger en la producción, adquisición y utilización de un texto escolar (Level y Mostacero, 2011; Ramírez, 2004). Para el caso venezolano, lo usual ha sido la utilización de textos producidos comercialmente mediante series, por año de estudio o nivel de los educandos.

Sobre la base de lo expresado más arriba acerca de que la escritura es una de las destrezas fundamentales en la enseñanza del idioma, de que las actividades son el componente más importante de cualquier curso, de que es aceptado que las concepciones de escritura pueden cambiar o mantenerse, de que los estudios con libros de texto pueden develar concepciones no siempre explícitas en ellos, así como de que es muy importante saber qué se enseña y qué se aprende, se ha decidido indagar cómo se aborda la escritura en libros de inglés de educación media y contribuir con este campo de la enseñanza. Por tanto, se ha llevado a cabo un estudio empírico con textos (Titscher, Meyer, Wodak y Vetter, 2000) con las siguientes preguntas de investigación:

¿Cuáles son las actividades de escritura del inglés que se promueven en libros de texto estatales de la educación media venezolana, específicamente de $4 .^{\circ}$ y $5 .^{\circ}$ año?

¿De qué manera se relacionan dichas actividades con las teorías actuales del campo de la enseñanza del inglés?

¿Qué tipo de soporte para el aprendizaje es probable que provean dichos textos a los educandos?

De tal modo, los objetivos del estudio son analizar las actividades de escritura que se promueven en dos libros de texto de inglés de $4 .^{\circ}$ y $5 .^{\circ}$ año, relacionar dichas actividades con las teorías actuales acerca del aprendizaje del inglés e indagar sobre qué tipo de soporte pudieran proveer dichos libros.

\section{Aspectos metodológicos}

\subsection{Criterios para la selección de los libros}

Los libros analizados son dos de la serie para la educación media My Fourth Victory y My Fifth Victory de $4 .^{\circ}$ y $5 .^{\circ}$ año respectivamente, con portada en color rojo, extensión de 208 páginas cada uno y dimensiones de un cuarto de pliego $(27 \times 21 \mathrm{~cm})$ en edición del año 2014. Fueron seleccionados de acuerdo con los criterios de ser de uso en instituciones del país, edición estatal con distribución gratuita y corresponder a $4 .^{\circ}$ y $5 .^{\circ}$ año. Para tal selección se razonó que si todos los libros de inglés se rigen por el mismo programa, entonces todos serán representativos de su nivel o ciclo. Se escogieron los dos últimos años de la Educación Media General debido a que es la etapa más avanzada de los educandos en la educación secundaria, antesala de su egreso hacia el nivel superior.

\subsection{Labor con los textos}

Los libros se organizan en unidades, de la unidad 1 a la 8 cada uno para un total de 16 unidades utilizadas en el estudio. Dichas unidades incluyen secciones que vehiculan las actividades referentes a las destrezas. Las secciones se diferencian por rectángulos a color, seriados mediante letras. Se presentan viñetas al final de cada unidad. Aquellas que contenían actividades de escritura se incluyeron en el estudio. Se excluyeron las actividades que no fueran de escritura del inglés. En total se consideraron para el análisis 
317 actividades de escritura -167 del libro 1 y 150 del 2- de un total de 609 secciones de actividades contenidas en los libros.

\subsubsection{Unidades de análisis}

Una lógica de coherencia metodológica ha guiado el presente estudio: si las actividades son desplegadas en estructuras del lenguaje entonces un análisis de cláusulas funcionará. De la lectura emergieron las unidades, que fueron las actividades de escritura presentes en los libros. Estas actividades son marcadas por cláusulas con un verbo rector. Las cláusulas son unidades gramaticales cuya estructura está constituida por las funciones sintácticas organizadas en torno a un verbo. Un ejemplo de cláusula de un libro es:

\section{Now answer these questions (libro 1, página 16)}

El verbo es central para el concepto de actividad pues es la categoría lingüística que expresa la acción, así como la existencia y la ocurrencia en la gran mayoría de las lenguas conocidas. Los verbos son muy importantes pues dan movimiento al significado y progresividad a la expresión (Palmer, 1978; Payne, 2004). La actividad de escritura se evidenció en diferentes niveles, desde rellenar y completar hasta redactar. Se excluyeron aquellas que no requerían el uso del signo lingüístico (p.e. mark, check, underline, entre otros) con, al menos, una palabra.

\subsubsection{Categorías de análisis}

Las cláusulas cuyos verbos son rectores -verbos en infinitivo en actos directivos o exhortativos- indican lo que el estudiante debe llevar a cabo en cada caso (por ejemplo: write, answer, complete) para un total de 317 categorías. Es importante notar que el verbo to complete, en sus dos acepciones aquí pertinentes siempre se relaciona con completar, Ilenar y rellenar. Por su parte, write es mucho más polisémico y presenta unas veinte acepciones relacionadas con tal actividad grafomotora, incluyendo la de completar.

En el siguiente cuadro figuran ejemplos de categorías, donde el primer número de la codificación de las cláusulas refiere al año de estudio $\left(4 .^{\circ}\right.$ o $\left.5 .^{\circ}\right)$ y el segundo al consecutivo de la actividad de escritura en el libro. Luego del guión, el tercer número corresponde a la página del libro a la que pertenece. La letra al final indica si la actividad refiere a $W=$ write, $A=$ answer,$C=$ complete u $\mathrm{O}=$ otra. La clasificación según estas cuatro letras obedece a la frecuencia de aparición de los verbos: los tres más frecuentes del total de 30 que se evidenciaron más una categoría que agrupara a los verbos restantes, la mayoría de ellos marginales, a excepción de fill in y translate con 23 y 18 casos respectivamente. Se estructuró de tal forma a fin de utilizar un modelo sencillo de cuatro partes. En dicho cuadro pueden verse ejemplos con los verbos más frecuentes. El verbo rector de la actividad de escritura aparece en negrita.

\section{Tabla 1. Ejemplos de unidades y categorías}

\begin{tabular}{|l|l|}
\hline $4^{\circ}{ }^{\circ}$ 5-13-W & In your notebook write questions to the following answers. \\
\hline $4^{\circ}$ 134-163-A & Read the following passage and answer the questions in your notebook. \\
\hline $5 .^{\circ} 38-49-\mathrm{C}$ & Complete the table with nouns from the text. \\
\hline $5 .^{\circ} 81-97-0$ & Now fill in the blanks with the words below. \\
\hline
\end{tabular}

Por razones metodológicas, los verbos fueron tomados según su función o valor gramatical dentro del co-texto de la cláusula. Pero, tal como se verá más adelante, este valor de los 
verbos no siempre referirá a su verdadera función pragmático-discursiva con relación a la actividad que en la práctica se requería de los estudiantes. Así, en las actividades de escritura de los libros analizados algunas veces tendrá más importancia el hacer que el ser.

\subsection{Estrategia de análisis}

De las 317 cláusulas emergió igual número de categorías de verbos, las que fueron colocadas en una tabla ad hoc. Se trata de una estrategia emergente con método de evaluación (Johnstone, 2000) en la que las categorías son suministradas por los propios textos al interactuar el investigador con ellos. Se diseñó una tabla de tres entradas, unidad por unidad, numeradas a la izquierda del 1 al 8 para cada libro. Se copiaron las actividades completas (167 para el libro 1 y 150 para el 2) en la segunda entrada de la tabla, con espacio a la derecha para comentarios. A cada actividad se le marcó en negrita el verbo rector tal como se ejemplificó más arriba.

Tabla 2. Actividades de escritura. Libro 1

\begin{tabular}{|l|ll|}
\hline Unidades & Actividades & Comentario/s \\
\hline $1-8$ & $1-167$ & ------------- \\
\hline
\end{tabular}

Tabla 3. Actividades de escritura. Libro 2

\begin{tabular}{|c|c|c|}
\hline Unidades & Actividades & Comentario/s \\
\hline $1-8$ & $1-150$ & |------------------- \\
\hline
\end{tabular}

\subsection{Procedimientos}

El análisis de las actividades según la frecuencia de su verbo rector llevó a configurar la clase de actividad. Las frecuencias fueron vertidas para cada uno de los libros en una tabla con cuatro entradas, según correspondiera a $\mathrm{W}=$ write, $\mathrm{A}=$ answer, $\mathrm{C}=$ complete $\mathrm{u} \mathrm{O}=$ otro en su función gramatical (ver tabla 4).

Tabla 4. Frecuencia de verbos en actividades de escritura en los dos libros

\begin{tabular}{|l|lll|}
\hline Write $(\mathrm{W})$ & Answer $(\mathrm{A})$ & Complete (C) & Otros (O) \\
\hline 141 & 48 & 40 & 91 \\
\hline
\end{tabular}

Se halló que el verbo más frecuente es write, con 141 casos de los 317 posibles; mientras que answer apareció en 48 casos y complete en 40. Los otros verbos son: fill in (23), translate (18), change (9), create (5), add (4), list (3), fill out (2), make (2), order (2), rewrite (2), say (2), write down (2) y continue, choose, copy, describe, design, elaborate, find, identify, organize, report, select, substitute, summarize y unscramble con un caso cada uno, para un total de 30 diferentes verbos. Vale resaltar que dos verbos, fill in (23) y translate (18) quedaron clasificados en la categoría de otros, pero tienen una figuración importante (41 sumando ambos). Tal como se mostrará en la sección siguiente, el análisis de las categorías produjo las clasificaciones de resultados, libro por libro. 


\section{Resultados}

\subsection{Libro 1 (4. ${ }^{\circ}$ año)}

En primer lugar se mostrará la importancia relativa que se asigna a la actividad de escritura en el libro de $4 .^{\circ}$ año (ver figura 1 ).

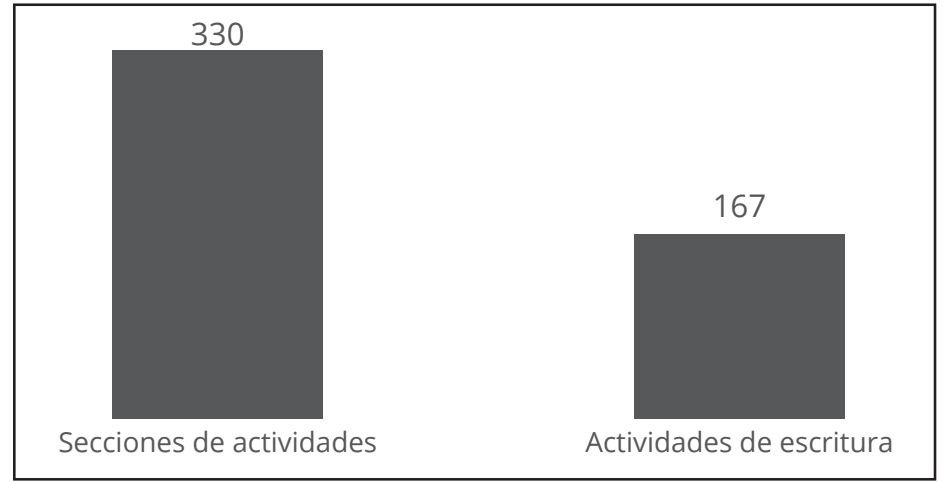

Figura 1. Actividades del libro 1 ( $4^{\circ}$ año)

En la figura 1 puede verse que en las 330 secciones que vehiculan las actividades del libro 1 se hallaron 167 actividades de escritura. Esto sugiere la importancia que se le otorga a dicha destreza. De manera no sorprendente el verbo to write es el que más frecuentemente rige actividades (77 veces). Pero yendo más allá, hacia la función pragmática de dicho verbo, se encontró que algo más de la mitad (40) de los casos refiere a actividades que implican trabajo con frases y oraciones, como en write questions to the following answers (p.70). No obstante, algo menos de la mitad (35) de los casos de write refiere a generación de textos, como en write a healthy menu for the day (p. 23). Esto sugiere una búsqueda de equilibrio y aunque no se prescinde del trabajo con frases y oraciones, sí se trata de promover la comunicación. Con respecto a esto, Brown (2007: 241) ha señalado que en la enseñanza comunicativa del idioma la labor del salón de clases no se restringirá a la competencia gramatical o lingüística; las actividades que en dicha enseñanza se diseñen serán para que los aprendices participen en el uso pragmático y funcional del idioma para propósitos significativos.

En la figura 2 puede verse la representación de las principales actividades de escritura que se promueven según las categorías de verbos.

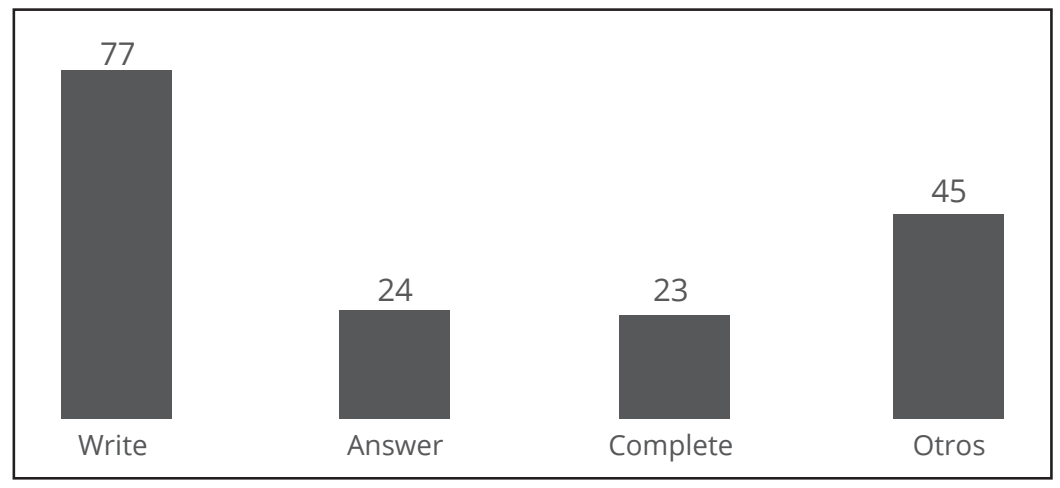

Figura 2. Verbos de las actividades del libro 1 (4ºño) 
La figura 2 indica que 77 de 167 -algo menos de la mitad- se corresponden con escribir, poco más de una de cada diez actividades con responder e igualmente con completar. Es de señalar que en la categoría otros quedó la actividad de rellenar (14 casos) que tiene casi uno de cada diez. Las actividades con otros verbos (45) hacen aproximadamente una de cada cuatro actividades. Una vez más, si examinamos más allá de la función gramatical de los verbos y vamos a la función pragmática, podrá verse que existen actividades de completar (complete) y de rellenar (fill in) para casos de escribir. Véase, por ejemplo, la tabla 5:

\section{Tabla 5. Ejemplos (libro 1)}

\begin{tabular}{|c|c|}
\hline $4^{\circ} 132-158-W$ & $\begin{array}{l}\text { In your notebook, write tag questions to the following } \\
\text { answers. }\end{array}$ \\
\hline $4 .^{\circ} 75-92-W$ & Complete with the appropriate verb form. \\
\hline
\end{tabular}

En el primer caso se trata en realidad de completar, por lo que en realidad sería complete. En el segundo se trata de colocar una sola palabra, por lo que se trataría entonces de fill in. De los verbos clasificados como otros son frecuentes fill in (14) y translate (8), como en los siguientes casos.

\section{Tabla 6. Ejemplos (libro 1)}

\begin{tabular}{|ll|}
\hline $4 .^{\circ}$ 154-185-O & $\begin{array}{l}\text { Fill in the blanks with to be in the past tense } \\
\text { and the correct form of the verb. }\end{array}$ \\
\hline $4 .^{\circ}$ 53-68-0 & $\begin{array}{l}\text { In your notebook translate the following } \\
\text { sentences into English. }\end{array}$ \\
\hline
\end{tabular}

Ambas son actividades frecuentes, con más de uno de cada diez casos.

Al relacionar las actividades de escritura con las teorías actuales acerca de la enseñanza del inglés, estos resultados sugieren que el libro se encuentra casi en equilibrio entre las concepciones estructuralistas, conductistas del aprendizaje, hoy día consideradas obsoletas, y un enfoque usualmente considerado más de avanzada, el comunicativo (Brown, 2007), aunque no haya unanimidad en un tópico tan complejo como el aprendizaje de idiomas en el que convergen múltiples variables locales (Bhowmik, 2015; Zeuch y Gregson, 2015).

Por un lado, de los enfoques que se centran en las estructuras del idioma, Hyland (2003) ha señalado que los pocos que hoy día siguen esta concepción asumen la escritura como una extensión de la gramática, una manera de reforzar los patrones del idioma por medio de la formación de hábitos o de evaluar las habilidades de los aprendices para producir oraciones bien estructuradas. Con este enfoque, que debería utilizarse mayormente en los niveles inferiores de los cursos, pueden generase serios problemas dado que la escritura está rígidamente controlada mediante tareas tales como rellenar, completar y transformar oraciones. En el mejor de los casos, lo más que podría lograrse sería la escritura guiada. La escritura libre queda por fuera (Hyland, 2003: 3-5). En cuanto a su componente estructuralista, el libro analizado podría tener la fortaleza de reconocer la preparación y el bagaje reales de los educandos (poco dominio del idioma) y tratar de no basarse en una única estrategia de escritura. Evidencia intentos por reflejar la manera en que las personas usan el idioma para comunicarse en situaciones auténticas (Hyland, 2003:95-97) (casi la mitad de las actividades con el verbo to write implican generación de textos). 
Por otro lado, en cuanto a qué tipo de soporte probablemente proveerá dicho libro a los educandos, del examen de los aspectos anteriores se puede colegir que el libro bien pudiera servir para ayudar a estructurar las lecciones que se imparten en ambientes problemáticos, con aulas superpobladas y necesidad de control de los grupos. Las actividades con generación de frases y oraciones, de rellenado y compleción, podrían mantener ocupados a los jóvenes educandos aunque sin necesariamente propiciar interacción la que, se sabe, es uno de los factores que promueven el aprendizaje significativo (Brown, 2007).

\subsection{Libro $2\left(5^{\circ}\right.$ año)}

En la figura 3 observamos la importancia otorgada a la escritura en el libro de $5 .^{\circ}$ año.

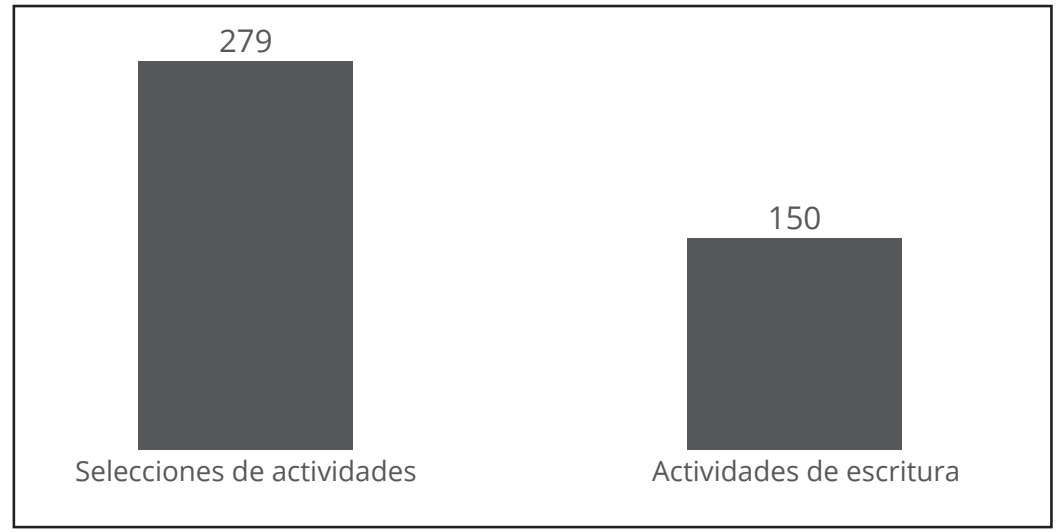

Figura 3. Verbos de las actividades del libro 2 ( $5^{\circ}$ año)

En la figura 3 se representa que, de manera poco diferente al libro 1, en las 279 secciones de actividades se hallaron 150 de escritura, lo cual sugiere la importancia que se otorga a esta destreza en el libro 2.

En lo referente a las actividades específicas de escritura que se promueven, en la figura 4 pueden verse los resultados según las categorías.

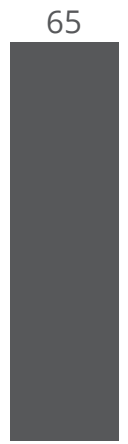

Write

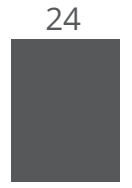

Answer

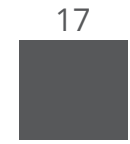

Complete

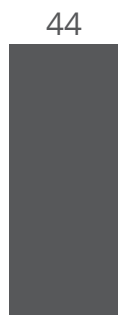

Otros

Figura 4. Verbos de las actividades del libro 2 ( $5^{\circ}$ año) 
Esta figura muestra que, nuevamente, los verbos write (65), answer (24) y complete (17) son los más frecuentes. Del rubro otros, igualmente translate (10) y fill in (9) son importantes. Hay casi igual presencia de otros verbos (44). Al igual que para con el libro anterior, si se examina más allá de la función gramatical de los verbos así como la función pragmática, estos resultados pudieran variar. Véanse los casos siguientes en las tablas 7 y 8.

\section{Tabla 7. Ejemplos (libro 2)}

\begin{tabular}{|ll|}
\hline $5 .^{\circ}$ 6-12-W & $\begin{array}{l}\text { Listen to your teacher carefully. Then write the missing } \\
\text { words. }\end{array}$ \\
\hline $5 .^{\circ}$ 24-34-C & $\begin{array}{l}\text { Listen and complete the sentences with the following } \\
\text { words. }\end{array}$ \\
\hline
\end{tabular}

Ambas son actividades de rellenado (fill in). Veamos ahora ejemplos con otros verbos.

\section{Tabla 8. Ejemplos (libro 2)}

\begin{tabular}{|ll|}
\hline $5^{\circ}$ 109-138-O & Preview the article and identify the following information. \\
\hline $5^{\circ} 131-163-0$ & $\begin{array}{l}\text { Reread the parts suggested and say what is being done: } \\
\text { comparing and contrasting, classifying, defining or argumenting. }\end{array}$ \\
\hline $5^{\circ}$ 77-88-0 & Listen to the words your teacher will say and write them down. \\
\hline
\end{tabular}

Son actividades, la primera y la segunda, de compleción (complete). La tercera es de dictado. Con estos ejemplos se busca significar que, mediante la función pragmática de los verbos, la naturaleza compleja del subsistema lexical del idioma puede jugar a favor de un componente estructuralista en las actividades. De ello no es fácil apartarse y puede suceder que a veces los libros sean en realidad más estructuralistas de lo que aparentan.

\section{Conclusiones y recomendaciones}

El estudio evidenció la importancia otorgada a la escritura en los libros analizados. Tales libros se encuentran casi en un equilibrio entre las concepciones estructuralistas, conductistas del aprendizaje, y otras como las comunicativas. Por una parte, dichos libros probablemente provean un soporte útil debido a que pudieran servir para ayudar a estructurar las lecciones en ambientes problemáticos, con aulas atestadas y necesitadas de control. Por otra, presentan actividades que implican la generación de textos. Los resultados en cuanto a concepciones estructuralistas y al tipo de lecciones tradicionales son consistentes con lo hallado por Beke (2015) y por Castillo, Gamero y Sivira (2015) en estudios con actividades de docentes de inglés de nuestro país. En cuanto a estrategias comunicativas, representan una innovación y un punto a favor de los libros. Con respecto a estos hallazgos vale decir que se evidencia, al menos parcialmente, la dificultad de que los avances de las ciencias, en este caso de la lingüística aplicada y la sociolingüística, ingresen completamente y con relativa prontitud al salón de clases promedio. Con relación a esto último se ha venido señalando desde hace décadas que con frecuencia son las variables políticas y socioeconómicas, entre otras (Abbott, 1992; Clarke, 1994; Giroux, 1988), las que suelen impedir que el progreso científico se traduzca en progreso educacional. Así, ha sido difícil estructurar en nuestro contexto, y de seguro en otros, actividades según el paradigma científico tenido como más avanzado, el comunicativo-funcional, el cual preconiza mayor libertad, más funcionalidad y menos estructuración. Así, el carácter de los libros analizados puede ser en parte un caso más del viejo principio de que en la historia de los estudios 
lingüísticos "la estructura no es la anécdota" (García y Vera, 1977: 24), por lo cual se podría decir que "esto es lo que hay" y que "hay que morir" con la estructura de la lengua.

Como se dijo, los libros analizados evidencian búsqueda de equilibrio entre dos corrientes contemporáneas de la teoría en cuanto a la enseñanza del idioma, y en ese particular quedan con suficientes haberes. No obstante, una preocupación que se ha manifestado con referencia a ciertas ediciones estatales (Montilla, 2015; Quintero, 2014; Ramírez, 2015)) tiene que ver con aspectos ideológicos, con propaganda y diseminación de contenidos que no se ajusten a la realidad. Aunque el análisis aquí realizado fue sintáctico, de actividades de escritura, no se pueden dejar de mencionar contenidos que llaman la atención y con los cuales cualquier venezolano informado estaría en desacuerdo. Algunos ejemplos, entre otros, son mensajes como los siguientes:

Libro 1: In Mérida we have the longest cable car of the world. Our cable runs from Mérida to the top of Pico Espejo (...) (p.124). Dicho teleférico, de gerencia estatal, ha permanecido cerrado desde 2008. (No lo tenemos, no circula)

Libro 2: During the 90s, State Venezuelan universities were becoming private, that is to say, some wanted to charge tuition fees, and there were few State universities. (p.130). Contenido no veraz. Se trata de un problema de déficit presupuestario de larga data, agravado desde 2008 (García Larralde, 2011).

Finalmente, hay que señalar un asunto. El hecho de que se coloquen tales o cuales actividades comunicativas, creativas (usualmente más exigentes) no significa que en la realidad se lleven a efecto. Esto tiene que ver, tal como lo señaló Beke (2015), con la necesidad de que se lleven a cabo estudios acerca del uso real y específico que se les da a los libros de inglés en las aulas venezolanas. Por último, es recomendable que los libros presenten contenidos ajustados a la realidad.

\section{Libros analizados}

My Fourth Victory Inglés. $4^{\circ}$ año. (2014). Caracas, Venezuela: Ministerio del Poder Popular para la Educación. Segunda Edición.

My Fifth Victory Inglés. $5^{\circ}$ año. (2014). Caracas, Venezuela: Ministerio del Poder Popular para la Educación. Segunda Edición.

\section{Referencias bibliográficas}

Abbott, G. (1992). Development, education and English language teaching. ELT Journal, 46(2), 172-179.

Apple, M. (1984). Economía política de la publicación de libros de texto. Revista de educación, (275) 43-62.

Arteaga, C. y Alemán, P. (2007). Representación del Caribe en los libros de texto de primaria venezolanos. Revista de pedagogía, 28(83), 335-360. Recuperado de http://www.scielo.org. ve/scielo.php?script=sci_arttext\&pid=S0798-97922007000300002\&lng=es\&tlng=es 
Baptista, T. (1910). Memoria que presenta el Ministro de Instrucción Pública al Congreso de los Estados Unidos de Venezuela. Tomo Primero. En: Fernández Heres, R. (1981) Memoria de cien años. Tomo IV. Volumen I, pp. 93-138. Caracas: Ediciones del Ministerio de Educación.

Beke, R. (2015). Inglés en la educación secundaria pública en Venezuela. En E. M. Zeuch y M. Gregson (coords.). La enseñanza del inglés en la escuela pública venezolana: evidencias, experiencias y perspectivas (pp. 47-76). Caracas: British Council/Universidad Central de Venezuela, Facultad de Humanidades y Educación, Comisión de Estudios de Postgrado.

Beyer, W. (2011). Constantes y variables en textos de matemática: un enfoque histórico. Paradigma, 32(2) 69-84. Recuperado de http://www.scielo.org.ve/scielo.php?script=sci arttext\&pid=S1011-22512011000200006\&lng=es\&tlng=es

Bhowmik, S. K. (2015) World Englishes and English Language Teaching: a pragmatic and humanistic approach. Colombian Applied Linguistics Journal. 17(1), 142-157. Recuperado de http://www.scielo.org.co/scielo.php?script=sci_arttext\&pid=S0123-46412015000100010

Bisbe, L. (2009). Huellas del racismo antiindígena en textos escolares venezolanos de finales de los años ochenta. Núcleo, 26, 11-35. Recuperado de http://www.scielo.org.ve/ pdf/nu/v21n26/art01.pdf

Bloch, M. (1979). Introducción a la historia. México: Fondo de Cultura Económica.

Briceño-Velazco, Y. (2014). "Aparentemente usted": postura y compromiso en editoriales de dos revistas venezolanas de gerencia. Signo y pensamiento [versión en línea], 33(65), 84-102. Recuperado de http://revistas.javeriana.edu.co/index.php/signoypensamiento/ article/view/11829/9694

Brown, D. H. (2007). Principles of language learning and teaching. White Plains, NY: PearsonLongman.

Castellanos, M.E. (2011). Una mirada al contexto y una propuesta para la universidad latinoamericana y venezolana en el presente siglo. En: La universidad venezolana en el siglo XXI. (pp. 25-40) Caracas: Universidad Católica Andrés Bello.

Castillo, E.; Gamero, M. y Sivira, Y. (2015). La práctica docente: concepciones de los profesores de inglés de secundaria. En E. M. Zeuch y M. Gregson (coords.). La enseñanza del inglés en la escuela pública venezolana: Evidencias, experiencias y perspectivas (pp. 179-187). Caracas: British Council/Universidad Central de Venezuela, Facultad de Humanidades y Educación, Comisión de Estudios de Postgrado.

Chaudron, C. (1998). Second language classrooms. Research on teaching and learning. Cambridge: Cambridge University Press.

Choppin, A. (2000). Los manuales escolares de ayer a hoy: el ejemplo de Francia. Historia de la educación, 19, 13-37. Recuperado de http://revistas.usal.es/index.php/0212-0267/ article/view/10790/11188

Ciapuscio, G. (2005). La noción de género en la lingüística sistémico funcional y en la lingüística textual. Signos 38(57), 31-48. Recuperado de http://www.redalyc.org/articulo. oa?id=157013764003 
Clarke, M. (1994). The dysfunctions of the theory/practice discourse. TESOL Quarterly, 28(1), 9-26.

Díaz Larenas, C.; Tagle, T.; Alarcón, P.; Quintana, M.; Ramos, L. y Vergara, J. (2013). Estudio de caso sobre las creencias de estudiantes de pedagogía en inglés respecto a la auto-eficacia percibida y la enseñanza del idioma. Núcleo, 30, 41-67. Recuperado de http://www.scielo. org.ve/scielo.php?script=sci_arttext\&pid=S0798-97842013000100002\&lng=es\&tlng=es

English First (2014). Índice de nivel de inglés [documento en línea]. Recuperado de http:// www.ef.co.ve/epi/

García, A. y Vera, A. (1977). Fundamentos de teoría lingüística. Málaga: Alberto Corazón Editor.

García Larralde, H. (2011). ¿Es viable el actual modelo financiero de las universidades? Líneas de cambio. En: La universidad venezolana en el siglo XXI. (pp. 163-178) Caracas: Universidad Católica Andrés Bello.

Gil Fortoul, J. (1912). Memoria que presenta el Ministro de Instrucción Pública al Congreso de los Estados Unidos de Venezuela. Tomo Primero. En: Fernández Heres, R. (1981) Memoria de Cien Años. Tomo IV. Volumen I, pp. 179-271. Caracas: Ediciones del Ministerio de Educación.

Giroux, H. (1988). Teachers as intellectuals: Toward a critical pedagogy of learning. Westport, CT: Bergin \& Garvey Publishers.

Gregson, M. (2006). English Next: ¿El fin del inglés como idioma extranjero? Núcleo, 23, 227-232. Recuperado de http://www.scielo.org.ve/scielo.php?script=sci_arttext\&pid=S079897842006000100010\&lng=es\&tlng=es

Güemes, R. (1994). Libros de texto y desarrollo del curriculum en el aula. Un estudio de casos. Tesis doctoral no publicada. Universidad de La Laguna, España. Recuperado de ftp://veda. bbtk.ull.es/ccssyhum/cs15.pdf

Guevara Rojas, F. (1915). Memoria que presenta el Ministro de Instrucción Pública al Congreso de los Estados Unidos de Venezuela. Tomo Segundo. En: Fernández Heres, R. (1981) Memoria de cien años. Tomo IV. Volumen I, pp. 439-577. Caracas: Ediciones del Ministerio de Educación.

Hernández, R. (2012). La enseñanza del inglés en Venezuela: Una visión retrospectiva. Heurística. Revista digital de historia de la educación [revista en línea], 15, 155-171. Recuperado de http://www.saber.ula.ve/bitstream/123456789/37306/1/articulo14.pdf

Herrera, D. (2012). Children as subjects with rights in EFL textbooks. Colombian Applied Linguistics Journal. 14 (1) 45-59. Recuperado de http://www.scielo.org.co/pdf/calj/v14n1/ $\underline{\mathrm{v} 14 \mathrm{n} 1 \mathrm{a} 04}$

Howatt, A. P. R. (1984) A History of English Language Teaching. Hong Kong: Oxford University Press.

Hyland, K. (2003). Second language writing. Nueva York: Cambridge University Press. 
Johnsen, E. B. (1996). Libros de texto en el caleidoscopio. Estudio crítico de la literatura y la investigación sobre los textos escolares. Barcelona: Pomares Corredor.

Johnstone, B. (2000). Qualitative methods in sociolinguistics. Nueva York: Oxford University Press.

Leal, I. (1981). Historia de la UCV. Caracas: Ediciones del Rectorado de la Universidad Central de Venezuela.

Level, M. B. y Mostacero, R. (2011). El texto escolar: ¿Artefacto didáctico? Investigación y postgrado, 26(2), 9-56. Recuperado de http://www.scielo.org.ve/scielo.php?script=sci arttext\&pid=S1316-00872011000200002\&lng=es\&nrm=iso\&tlng=es

Lopes, C. (2015). Livro didático e a pedagogia do mestre ignorante. Trabalhos em Linguística Aplicada. 54 (2) 223-243. Recuperado de https://dx.doi.org/10.1590/010318134607166141

López de D’Amico, R. (2010). La enseñanza del inglés en Venezuela. En: I. Cantón, R. Valle, A. Arias, R. Baelo y R. Cañón (coords.). Retos educativos en la sociedad del conocimiento (pp. 392-401). Barcelona: Davinci Continental.

López de D’Amico, R. (2015). La formación de docentes de inglés. En E. M. Zeuch y M. Gregson (coords.). La enseñanza del inglés en la escuela pública venezolana: Evidencias, experiencias y perspectivas (pp. 77-107). Caracas: British Council/ Universidad Central de Venezuela, Facultad de Humanidades y Educación, Comisión de Estudios de Postgrado.

Medina, A. y Domínguez, C. (2009). La novela por entregas como género discursivo. El caso de The way we live now de Anthony Trollope. Núcleo. 26: 201-224. Recuperado de http://www. scielo.org.ve/pdf/nu/v21n26/art08.pdf

Montilla, A. (2015). Libros del gobierno son el texto único en escuelas oficiales. El Nacional. Sociedad. Recuperado de http://www.el-nacional.com/sociedad/Libros-gobierno-textoescuelas-oficiales_0 557344441.html

Mota, C. (2006). El rol de la escritura dentro del currículo de la enseñanza y aprendizaje del inglés como segunda lengua (esl/efl): Una perspectiva histórica. Acción Pedagógica, 15, 56-63. Recuperado de http://www.saber.ula.ve/bitstream/123456789/17280/2/articulo6.pdf

Mujica, B.; Díaz, L. y Arnáez, P. (2008). Concepción de escritura en los libros de texto de educación básica. Revista de Pedagogía, 29(85), 269-289. Recuperado de http://www.scielo. org.ve/scielo.php?script=sci_arttext\&pid=S0798-97922008000200004\&lng=es\&nrm=iso\&t $\underline{\operatorname{lng}=e s}$

Palmer, F. R. (1978). The English verb. Whitstable, Reino Unido: Longman.

Payne, T. (2004). Describing morphosyntax. A guide for field linguistics. Cambridge: Cambridge University Press.

Puéllez, M. y Tiana, A. (2003). El proyecto MANES: Una investigación histórica sobre manuales escolares. Boletín de la Institución Libre de Enseñanza, 49-50, 163-174. 
Quintero, I. (2014) Enseñar Historia: 7 anotaciones sobre la Colección Bicentenario. Prodavinci. Recuperado de http://prodavinci.com/2014/05/27/actualidad/ensenar-historia7-anotaciones-sobre-la-coleccion-bicentenario-por-ines-quintero/

Raimes, A. (1983). Tradition and revolution in ESL teaching. TESOL Quarterly, 17(4), 535-552.

Raimes, A. (1998). Teaching writing. Annual Review of Applied Linguistics, 18, 142-167.

Ramírez, T. (2004). El texto escolar en el ojo del huracán. Caracas: Universidad Central de Venezuela, Fondo Editorial Facultad de Humanidades y Educación.

Ramírez, T. (2007). Del control estatal al libre mercado. Políticas Públicas y textos escolares en Venezuela (1958-2005). Caracas: Ediciones de la Biblioteca de la Universidad Central de Venezuela.

Ramírez, T. (2015) ¿Enseñar historia o reconstruir la historia? Los textos escolares de Ciencias Sociales de la Colección Bicentenario. Conferencia presentada en la Academia Nacional de la Historia, Caracas, 5 de diciembre de 2015.

Rodrigues, P. y Marques da Fonseca, E. (2014). A produção textual no livro didático do ensino médio: Identificação da abordagem teórica. Acta Scientiarum. Language and Culture [versión en línea], 36(4), 427-435. Recuperado de http://www.redalyc.org:9081/articulo. oa?id $=307432548008$

Savignon, S. (2005). Communicative language teaching: Strategies and goals. En: E. Hinkel (coord.). Handbook of research in second language teaching and learning (pp. 635-651). Mahwaj, NY: Lawrence Erlbaum.

Titscher, S.; Meyer, M.; Wodak, R. y Vetter, E. (2000). Methods of text and discourse analysis. Londres: Sage.

Tosi, C. (2011). El texto escolar como objeto de análisis. Un recorrido a través de los estudios ideológicos, didácticos, editoriales y lingüísticos. Lenguaje. 39 (2) 469-500. Recuperado de http://www.scielo.org.co/pdf/leng/v39n2/v39n2a08.pdf

Varón, M. (2009). Componente cultural, libros de texto y enseñanza del inglés como lengua extranjera. Forma y función. 22 (1) 95-124. Recuperado de http://www.redalyc.org/articulo. oa?id=21912427004

Villar, J. (2012). Venezuela: Medalla de bronce entre los que peor hablan inglés [documento en línea]. Recuperado de http://periodismo3ucv.blogspot.com.uy/2012/04/venezuelahabla-ingles-tan-mal-como-lo.html

Webb, D.; Metha, A.: y Jordan, F. (1996). Foundations of American education. Englewood Cliffs, NJ: Merrill Prentice Hall.

Wray, A. y Bloomer, A. (2006). Projects in linguistics. A practical guide to researching language. Londres: Hodder Arnold. 
Zapata, C. (2011). Estrategias de comprensión lectora en libros de inglés como lengua extranjera. Educare, 15(3), 27-52. Recuperado de http://revistas.upel.edu.ve/index.php/ educare/article/viewFile/580/210

Zeuch, E. M. y Gregson, M. (2015). (Coords.). La enseñanza del inglés en la escuela pública venezolana: evidencias, experiencias y perspectivas. Caracas: British Council/Universidad Central de Venezuela, Facultad de Humanidades y Educación, Comisión de Estudios de Postgrado. Recuperado de http://englishagenda.britishcouncil.org/sites/ec/files/F145\%20 Venezuela_combined_FINAL_V2.pdf

(*) Estudio producido dentro de la línea de investigación El texto escolar como objeto de investigación del Doctorado en Educación de la Universidad Central de Venezuela y el Centro de Investigaciones Educativas (CIES) de dicha universidad. 